\title{
ARTICLE OPEN \\ Coculture of meniscus cells and mesenchymal stem cells in simulated microgravity
}

\author{
William M. Weiss ${ }^{1,2}$, Aillette Mulet-Sierra ${ }^{1}$, Melanie Kunze ${ }^{1}$, Nadr M. Jomha $^{1}$ and Adetola B. Adesida (D)
}

Simulated microgravity has been shown to enhance cartilaginous matrix formation by chondrocytes and chondrogenesis of mesenchymal stem cells (MSCs). Similarly, coculture of primary chondrocytes with MSCs has been shown as a strategy to simultaneously retain the differentiated phenotype of chondrocytes and enhance cartilaginous matrix formation. In this study, we investigated the effect of simulated microgravity on cocultures of primary human meniscus cells and adipose-derived MSCs. We used biochemical, qPCR, and immunofluorescence assays to conduct our investigation. Simulated microgravity significantly enhanced cartilaginous matrix formation in cocultures of primary meniscus cells and adipose-derived MSCs. The enhancement was accompanied by increased hypertrophic differentiation markers, COL10A1 and MMP-13, and suppression of hypertrophic differentiation inhibitor, gremlin 1 (GREM1).

npj Microgravity (2017)3:28 ; doi:10.1038/s41526-017-0032-x

\section{INTRODUCTION}

The menisci of the knee are a pair of fibrocartilaginous tissues. ${ }^{1}$ They primarily serve as mechanical load distributors within the knee joint. ${ }^{2}$ Traumatic tears in the avascular region of the tissue are common and do not heal. ${ }^{1}$ Treatment options for these tears are currently limited to partial meniscectomy. ${ }^{3,4}$ However, partial meniscectomy is a major risk factor for the early development of knee osteoarthritis. ${ }^{3,4}$ Tissue engineering using cells presents a potential option to create functional tissues to replace damaged meniscus. $^{5-15}$ Primary meniscus cells (MC) are the ideal cell sources as they closely resemble the in vivo phenotype of $M C$ and can form the biomechanically functional extracellular matrix (ECM) of the meniscus. ${ }^{1,16,17}$ Obtaining sufficient numbers of primary MC for meniscus tissue engineering is often impossible and impractical. $^{18,19}$ To circumvent this limitation, primary MC are expanded in in vitro culture. However, expanded MC suffer from dedifferentiation and loss of the functional matrix-forming phenotype of native $M C^{18,19}$ Supplementation of primary MC through direct coculture with bone marrow mesenchymal stem cells (MSCs) has been demonstrated as a strategy to retain the differentiated phenotype of primary $M C$ with the additional benefits of synergistic production of the functional matrix components of the meniscus ${ }^{20-22}$ and downregulation of hypertrophic differentiation of MSCs. ${ }^{21,23}$ However, the use of bone marrow MSCs potentially presents another anatomical site other than the knee for MSCs harvest. Supplementation of primary MC with knee infrapatellar fat pad derived MSCs may be a better anatomical option.

The differentiation of multiple types of MSCs is influenced by a wide range of biochemical, ${ }^{24}$ microenvironmental, ${ }^{25}$ and mechanical factors. ${ }^{26-28}$ TGF- $\beta 1$ and TGF- $\beta 3$, members of the TGF- $\beta$ superfamily, that promote fibro-chondrogenic differentiation of $\mathrm{MSCs}^{29}$ and production of meniscus-like ECM. Diverse types of mechanical stimuli, including compressive load $^{26}$ simulated microgravity (SMG), ${ }^{30}$ low-intensity pulsed ultrasound, ${ }^{27,28,31}$ and hydrostatic pressure has been reported to induce or regulate the differentiation of MSCs through TGF- $\beta$ signaling pathway. ${ }^{32}$ Hypoxia stimulated chondrogenic differentiation of bone marrow-derived MSCs by induction of TGF- $\beta 1$ gene expression and protein production. ${ }^{33}$

The rotary cell culture system (RCCS) bioreactor, developed by the National Aeronautics and Space Administration (NASA), as a tool to simulate microgravity has been shown to provide a relatively well-defined fluid dynamic environment with efficient mass transfer for nutrients and gases and low shear stress for tissue growth. ${ }^{34}$ RCCS has been reported to produce hydrodynamic forces supporting the development of tissue structures resembling cartilage ${ }^{35,36}$ and meniscus. ${ }^{37}$ Simulated microgravity facilitated the retention of the differentiated phenotype of chondrocytes within 3D porous scaffolds. ${ }^{36}$ Moreover, RCCS synergistically enhanced TGF- $\beta 1$-mediated chondrogenesis of human adipose-derived MSCs. ${ }^{30}$ However, to the best of our knowledge, no studies has investigated the effect of SMG on TGF$\beta$-mediated chondrogenesis in cocultures of primary human MC and infrapatellar fat pad-derived MSCs.

In the present study, primary human MC were cocultured with infrapatellar fat pad-derived MSCs on a 3D porous collagen scaffold in the presence of TGF- $\beta 3$, and cultured under SMG using the RCCS bioreactor. The goals of the present study were (a) to investigate the effect of SMG on human MC, (b) to determine the effect of SMG on the interaction of MC and MSCs in coculture and (c) to assess the potential benefit of using SMG to tissue engineer meniscus constructs in 3D porous type I collagen scaffolds. We hypothesized that SMG will augment the synergistic interaction between primary human MC and MSCs, and result in increased matrix production with suppression of hypertrophic differentiation.

\footnotetext{
${ }^{1}$ Laboratory of Stem Cell Biology and Orthopaedic Tissue Engineering, Department of Surgery, University of Alberta, Li Ka Shing Centre for Health Research Innovation,

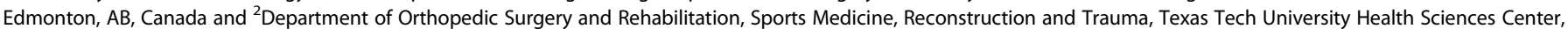
Lubbock TX USA

Correspondence: Adetola B. Adesida (adesida@ualberta.ca)
}

Received: 13 May 2017 Revised: 17 September 2017 Accepted: 22 September 2017

Published online: 10 November 2017 


\section{RESULTS}

Colony forming characteristics and immuno-phenotype of adipose stem cells (ASC)

A proportion of the seeded adipose tissue-derived mononucleated cells (MNCs) formed plastic adherent cell populations with distinct colonies. The developed colonies stained with crystal violet (Fig. 1a). Microscopic visuals of the colonies revealed cells with fibroblastic morphologies characteristics of plastic adherent
MSCs (Fig. 1b). We determined the clonogenicity of the MNCs to be $10.65 \pm 4.15 \%$ (Fig. 1c). The adherent cell populations were positive for the panel of cell surface markers characteristic of MSCs; ${ }^{38,39}$ CD13, CD29, CD44, CD73, CD90, CD105, and CD151 (Fig. $1 \mathrm{~d})$. Their relative mean fluorescence intensities (MFI) $\pm \mathrm{SD}$ are as in the parentheses: CD13 (29.26 \pm 10.79$), C D 29$ (2.83 \pm 0.35$), C D 44$ $(6.40 \pm 2.40)$, CD73 (8.63 \pm 3.79$),$ CD90 (113.62 \pm 14.90$), C D 105$ $(1.87 \pm 0.05)$, and CD151 (8.57 \pm 0.16$)$-Fig. 1e. The proportion (\%)
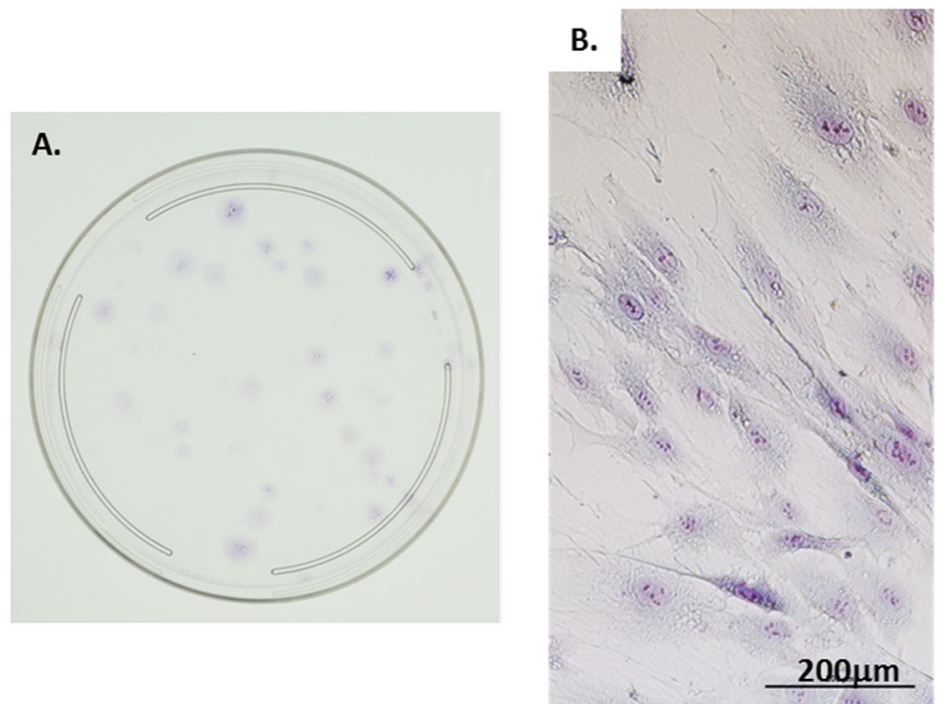

C.

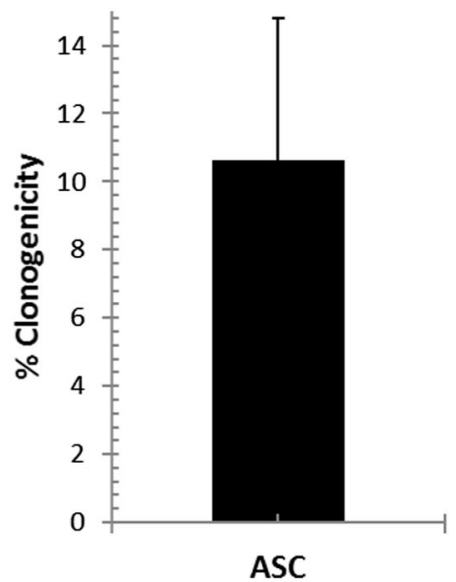

D.

CD13

CD29

CD34

CD44

CD45
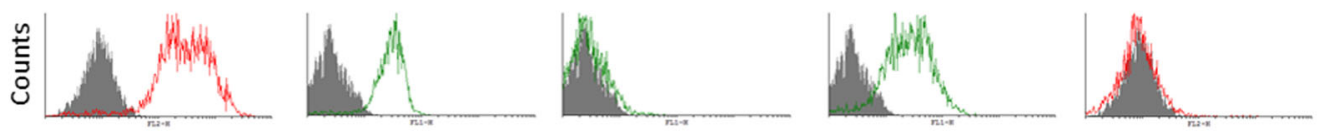

\section{CD73}

\section{CD90}

CD105

CD151

CD184
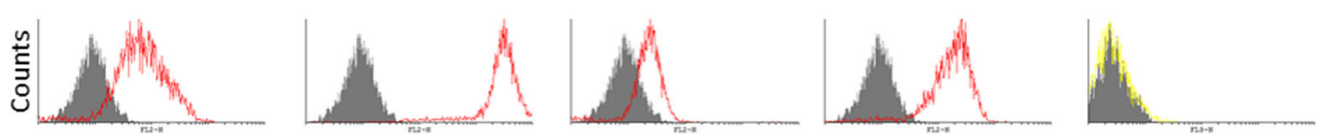

E.
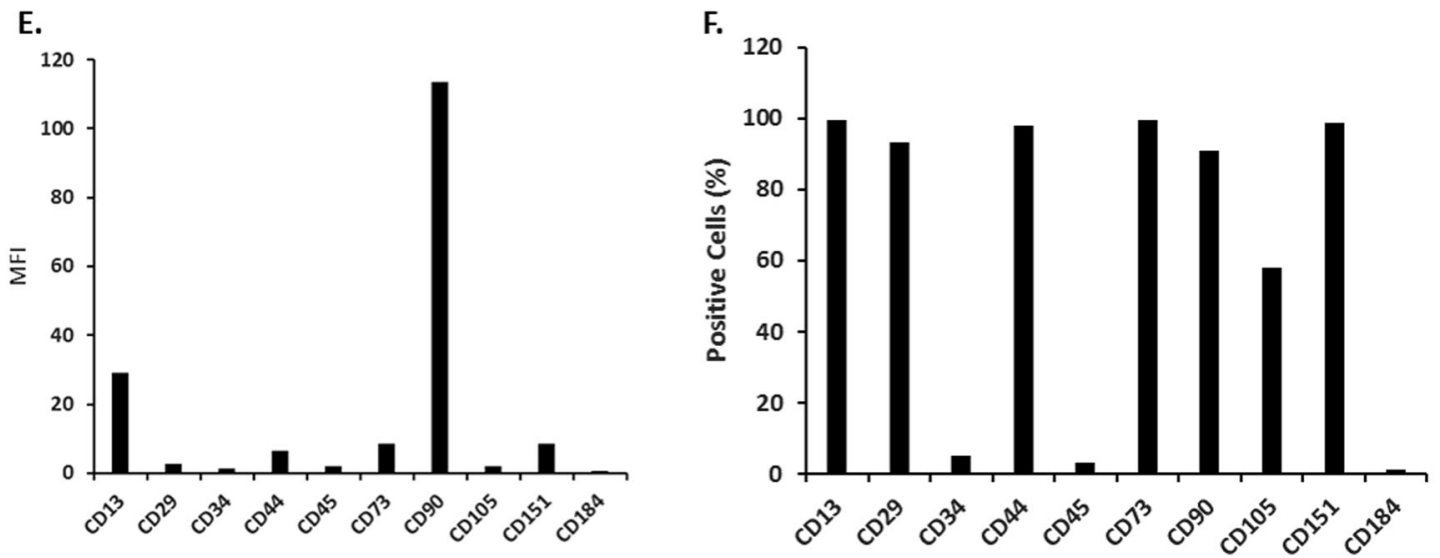

Fig. 1 Colony-forming unit fibroblastic characteristic and surface marker phenotype of fat pad-derived cells following tissue culture plastic adherent culture expansion. a A digital photograph of a CFU-F assay Petri dish after staining with crystal violet. b Representative photomicrographs of adherent cells from a 40-year-old male at passage (P0). c Proportion (\%) of total fat pad-derived MNCs seeded forming cell colonies (\% clonogenicity). d Surface marker phenotype of fat pad-derived adherent cells following culture expansion until passage 2 (P2). For each CD marker tested, solid histograms show negative isotype control; open histograms show CD marker expression. e Represents the mean fluorescence intensity (MFI) of two donors. f Proportion of positive (\%) cells expressing tested CD markers 
of cells that were positive for these markers varied considerably; CD13 (99.67 \pm 0.15$),$ CD29 (93.18 \pm 3.26$),$ CD44 (98.06 \pm 0.08$),$ CD73 $(99.67 \pm 0.09), \operatorname{CD} 90(90.97 \pm 8.86), C D 105$ (57.94 \pm 11.38$)$, and CD151 (98.86 \pm 0.16$)$, an indication of heterogeneity (Fig. 1f). In contrast to the MFI associated with mesenchymal markers, the cells displayed lower MFIs for markers of hematopoietic lineage; CD34 (1.29 \pm 0.02$)$ and CD45 (1.92 \pm 0.50$)$. The proportion (\%) of cells that were positive for CD34 and CD45 were $4.93 \pm 0.39$ and $3.16 \pm 1.10$, respectively. Interestingly, the cells had a low MFI $(0.73 \pm 0.52)$ for the $C-X-C$ chemokine receptor type 4 (CXCR-4) also known as CD184, which has been reported to be expressed on synovial fluid-derived MSCs. ${ }^{40}$ The proportion of $\mathrm{CD}_{184^{+}}$cells was $1.29 \pm 0.05 \%$.

Fluorescent-labeled cells reveal coculture of primary MC and ASC Pure ASC and pure MC were labeled successfully with cell membrane fluorescent dyes PKH26 (red; Fig. 2a) and PKH67 (green; Fig. 2b), respectively. Pure ASC (Fig. 2c), pure MC (Fig. 2d), and cocultured cells at $25 \% \mathrm{MC}$ and $75 \%$ ASC (Fig. 2e) retained their respective fluorescent dyes after 4 weeks of chondrogenic culture in type I collagen matrix scaffolds. This aspect of the study was performed only under static conditions.

Chondro-induction is enhanced in SMG cocultures of primary MC and ASC

Cell-seeded scaffold constructs of pure MC, pure ASC, and cocultures of MC and ASC were cultured in serum-free chondrogenic media for up to 28 days in the presence of TGF- $\beta 3$ under static or SMG conditions. Qualitative histological analysis of the constructs with safranin $\mathrm{O}$ staining demonstrated intense positive glycosaminoglycan (GAG) staining after the 28 days of culture under static and SMG conditions (Fig. 3). All groups were intensely positive for safranin $\mathrm{O}$ and nuclei content, an indication of significant synthesis and deposition of sulfated GAG matrix in the constructs (Fig. 3a-h). However, the deposition of GAG matrix appears to be more homogenously distributed in the constructs cultured under SMG (Fig. 3e-h). Except for pure MC constructs, the characteristic phenotype of hypertrophic chondrocytes of enlarged size/lacunae was evident in groups containing ASC. Quantitative analysis for the GAG contents of the constructs via DMB assay demonstrated that GAG contents were higher in constructs containing cocultured cells regardless of whether the constructs were cultured under static or SMG conditions (Fig. 4a). The constructs formed under static conditions from the coculture of $25 \% \mathrm{MC}$ and $75 \%$ ASC had a measured GAG content of 195.25 $\pm 38.73 \mu \mathrm{g}$ (mean \pm SD) compared to an expected GAG content of $148.50 \pm 38.36 \mu \mathrm{g}$. Wilcoxon signed rank pairwise comparison test between the measured and expected GAG quantities confirmed a significantly higher measured GAG content than the expected values $(p=0.003$; Fig. $4 a)$. Similarly, there were significant differences between the measured and expected GAG content of cocultured constructs containing $25 \% \mathrm{MC}$ and $75 \%$ ASC after culture under SMG. The SMG cultured constructs had a measured GAG content of $279.39 \pm 124.09 \mu \mathrm{g}$ relative to an expected GAG content of $167.78 \pm 75.39 \mu \mathrm{g}$. Pairwise comparison test using Wilcoxon signed rank test revealed a significant difference between the measured and expected GAG values ( $p=0.003$; Fig. 4a). After static culture, the constructs containing 50\% MC and $50 \%$ ASC had a measured GAG content of $190.53 \pm 47.84 \mu \mathrm{g}$. The expected GAG values for these constructs were $172.21 \pm 54.80 \mu \mathrm{g}$. However, there was no significant difference between the measured and expected GAG values as per Wilcoxon signed rank test $(p=0.109 ;$ Fig. 4a). In contrast, after SMG culture, the constructs containing $50 \% \mathrm{MC}$ and $50 \%$ ASC measured a significantly different GAG content of $251.63 \pm 121.92 \mu \mathrm{g} \mathrm{com}-$ pared to an expected GAG content of $173.04 \pm 62.29$. The $p$-value for the pairwise comparison was 0.026 as per Wilcoxon signed rank test (Fig. 4a).

After calculating the interaction index as a ratio of the measured and expected GAG contents of the constructs containing cocultured cells, we confirmed that the interaction indices were greater than 1, signifying that chondro-induction had occurred (Fig. 4b). Shapiro Wilk test for normality of data distribution proved positive. The Levene's test was significant and lacked homogeneity of variances. For cocultured cells at $25 \% \mathrm{MC}$ and $75 \%$ ASC, the interaction index was $1.35 \pm 0.16$ after culture under static conditions and $1.72 \pm 0.39$ after culture under SMG (Fig. 4b). Paired one-tailed Student's $t$ test (with unequal variance) comparison of the interaction indices revealed a significantly higher chondro-induction under SMG conditions $(p=0.006$; Fig. 4b). For cocultured cells at $50 \% \mathrm{MC}$ and $50 \%$ ASC ratio, the interaction index was $1.16 \pm 0.14$ after static culture and $1.49 \pm$ 0.44 after SMG culture. Similarly, the level of chondro-induction after SMG culture conditions was significantly higher $(p=0.022$; Fig. 4b).

\section{Simulated microgravity increases DNA content in MC and} cocultures

We next explored the possibility that cell proliferation may accompany the enhanced chondro-induction in cocultures of MC and ASCs after culture under SMG. Shapiro Wilk test for DNA content distribution proved to be normal. However, the Levene's test for homogeneity of data variance proved negative. To that end, a paired one-tailed Student $t$ test with unequal variance was used to assess differences between parallel static and SMG groups. First, we compared the DNA contents of constructs containing pure MC and pure ASC after static culture and SMG culture conditions. The DNA content of constructs containing pure MCs was $4.53 \pm 0.81 \mu \mathrm{g}$ after SMG culture compared to $3.73 \pm 0.63 \mu \mathrm{g}$ after static culture. These values were significantly different $(p=0.03$; Fig. $4 c)$. In contrast, the DNA content of constructs containing pure ASCs after static culture and SMG culture approached significant difference $(p=0.05)$. The DNA contents of pure ASC containing constructs after static culture and SMG culture were $3.35 \pm 0.56 \mu \mathrm{g}$ and $2.67 \pm 0.84 \mu \mathrm{g}$, respectively (Fig. 4c). The constructs containing $25 \% \mathrm{MC}$ and $75 \%$ ASC had a DNA content of $5.46 \pm 2.11 \mu \mathrm{g}$ after SMG culture and $4.20 \pm 0.57 \mu \mathrm{g}$ after static culture. These values were not significantly different $(p=0.08)$ (Fig. 4c). In contrast, constructs containing 50\% MC and 50\% ASC were significantly different $(p=0.03)$ in DNA contents after static culture and SMG culture. The constructs had a DNA content of 5.33 $\pm 1.49 \mu \mathrm{g}$ after SMG culture and those after static culture had a DNA content of $4.06 \pm 0.41 \mu \mathrm{g}$ (Fig. $4 \mathrm{c}$ ).

Effect of SMG on gene expression of chondrogenically stimulated MC, ASC, and cocultures of MC and ASCs

We used quantitative polymearse chain reaction (qPCR) to investigate the gene expression of chondrogenically stimulated MC, ASC and cocultures of MC and ASCs after static and SMG culture conditions. Data followed normal distribution as assessed by Shapiro Wilk test. However, the Levene' assessment of error variance proved highly significant. To this end, pairwise comparison between gene expression data of static and SMG cultured constructs were performed using paired Student's $t$ test with unequal variance.

SMG enhanced the mRNA expression of ACAN by 1.9 -fold in pure MCs $(p=0.0002$; Fig. 5a). SMG increased the mRNA expression of $A C A N$ by 2.2 -fold in pure ASC ( $p=0.036$; Fig. $5 a$ ). SMG enhanced the expression of $A C A N$ by 2.4 -fold in cocultures of $25 \%$ MC and $75 \%$ ASC $(p=0.0001$; Fig. 5a). Similarly, SMG enhanced the mRNA expression of ACAN by 3.6-fold in $50 \%$ MC and $50 \%$ ASC cocultures (Fig. 5a). In contrast, the mRNA expression of COL1A2 remained unperturbed between static and SMG cultured groups; no fold changes in COL1A2 expression were 

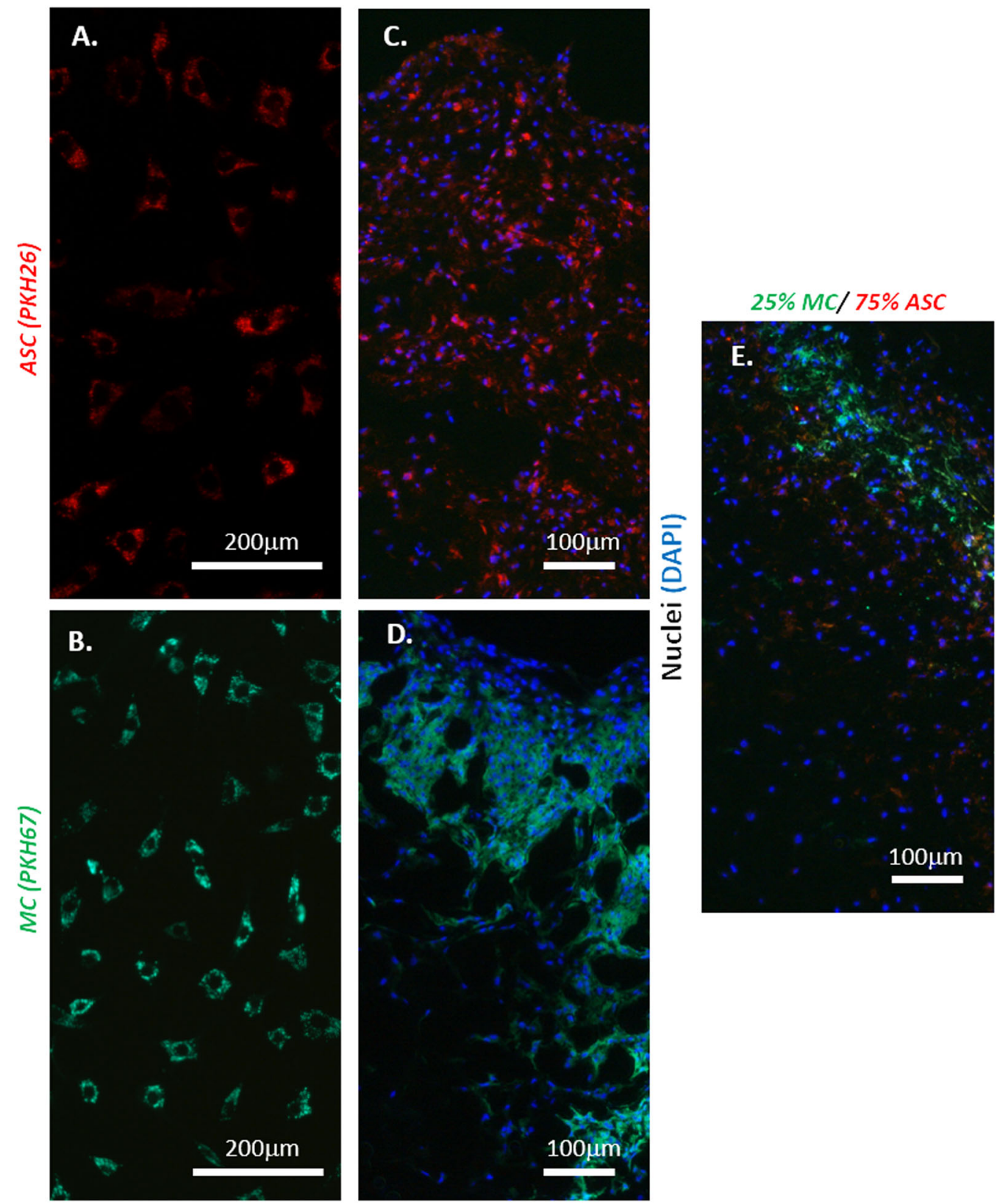

Fig. 2 Fluorescence photomicrographs of PKH26 and PKH67 labeled cells in monolayer and in three-dimensional (3D) porous scaffolds. a Representative fluorescence photomicrograph of passage 2 (P2) fat pad-derived plastic adherent cells labeled with PKH26 (red) cell membrane dye (male, 19 years old). Cells were labeled in suspension and imaged after $30 \mathrm{~min}$ of plating in monolayer. b Representative fluorescence photomicrograph of primary human meniscus cells labeled with PKH67 (green) cell membrane dye (male, 56 years old). Cells were labeled in suspension and imaged after $30 \mathrm{~min}$ of plating in monolayer. c Representative fluorescence photomicrograph of passage 2 (P2) fat padderived plastic adherent cells (ASC) labeled with PKH26 (red) cell membrane dye (male, 19 years old) after 3 weeks of chondrogenic culture in porous type I collagen scaffold. Cells were visualized after sectioning at $7 \mu \mathrm{m}$ and additional staining with DAPI for cell nuclei identification. $\mathbf{d}$ Representative fluorescence photomicrograph of primary human meniscus cells (MC) labeled with PKH67 (green) cell membrane dye (male, 56 years old) after 3 weeks of chondrogenic culture in porous type I collagen scaffold under static condition. Cells were visualized after sectioning at $7 \mu \mathrm{m}$ and additional staining with DAPI for cell nuclei identification. e Representative fluorescence photomicrograph of cocultured primary human meniscus cells (MC) labeled with PKH67 (green) and passage 2 (P2) fat pad-derived plastic adherent cells (ASC) labeled with PKH26 (red) after 3 weeks of chondrogenic culture in porous type I collagen scaffold under static conditions. The cells were premixed at a cell ratio of $25 \%$ MC and $75 \%$ ASC prior to seeding a total of 250,000 cells per type I collagen scaffold. Cells were visualized after sectioning at $7 \mu \mathrm{m}$ and additional staining with DAPI for cell nuclei identification. Scale bars: A and B $(200 \mu \mathrm{m})$ and C-E $(100 \mu \mathrm{m})$

observed. The insignificant differences in the treatment groups were supported by $p$-values $>0.05$ (Fig. 5b). SMG increased the expression of COL2A1 in pure MC, pure ASC, and in the cocultures of these cells. However, the fold increase observed were not statistically significant; SMG increased COL2A1 mRNA expression by: 1.2 -fold in pure $M C(p=0.28), 1.7$-fold in pure ASC $(p=0.16)$, 1.4-fold in cocultures of $25 \% \mathrm{MC}$ and $75 \%$ ASC $(p=0.06)$, and 1.2fold in cocultures of $50 \%$ MC and $50 \%$ ASC $(p=0.21)$. Qualitative immunofluorescence detection confirmed the translation of these collagen genes in the constructs (Fig. 6). All groups were positive for type I and II collagen. No notable differences were evident in the deposition of these collagens between static culture and SMG 

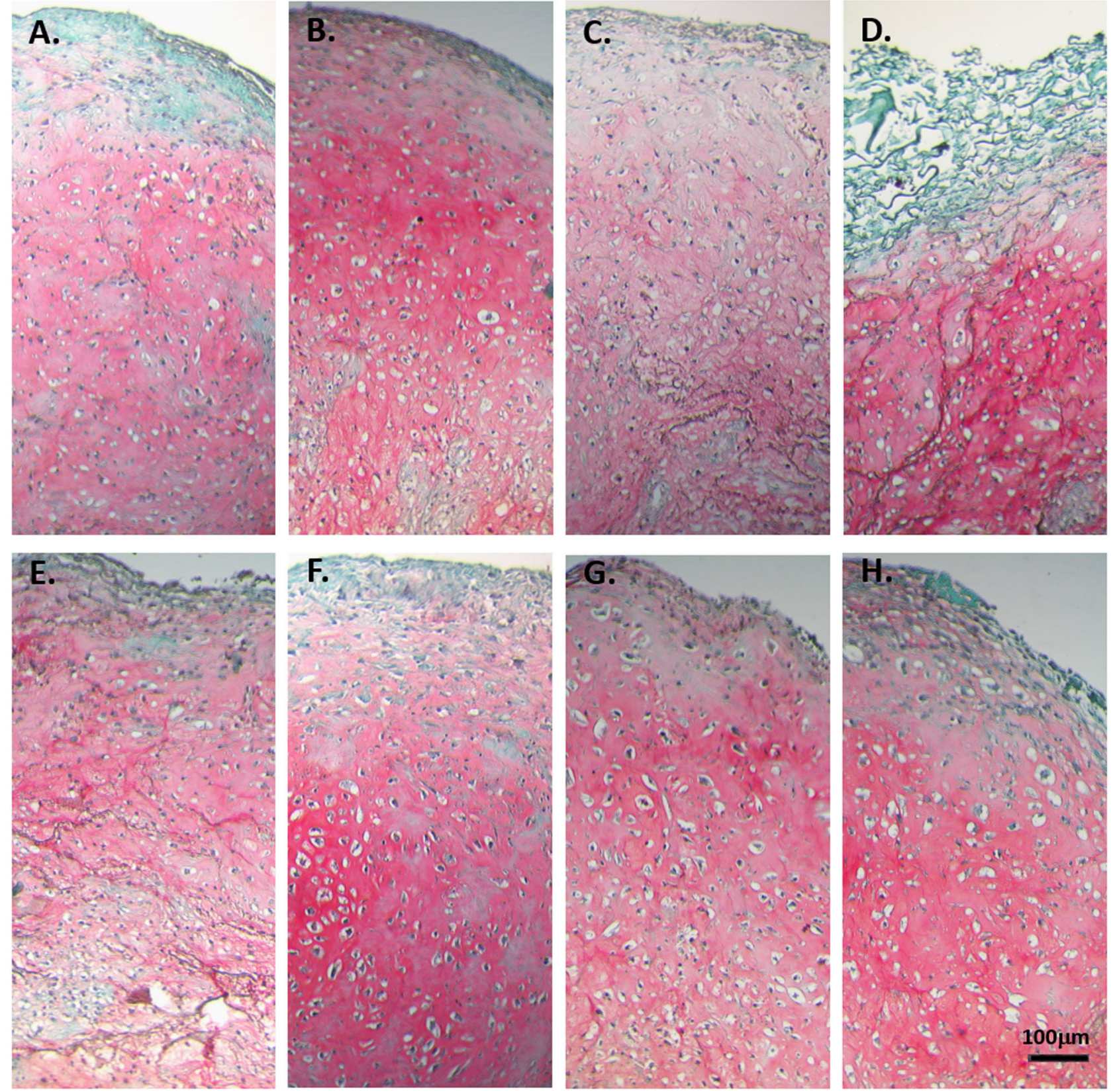

Fig. 3 Histological Safranin O staining after chondrogenic stimulation and differentiation of primary human meniscus cells and fat padderived adipose stem cells in porous type I collagen scaffolds. Representative safranin $\mathrm{O}$ staining of cell-scaffold constructs after 4 weeks of culture in a defined serum-free chondrogenic medium containing transforming growth factor- $\beta 3$ (TGF $\beta 3$ ) and dexamethasone (DEX) under static a-d and simulated microgravity e-h conditions: a, e pure primary human meniscus cells (MCs; male, 56 years); $\mathbf{b}$, f Passage 2 (P2) fat padderived adipose stem cells (ASC; male, 19 years); c, $\mathbf{g}$ Coculture of $75 \%$ (P2) fat pad-derived adipose stem cells (ASC; male, 19 years) with $25 \%$ primary human meniscus cells (MCs; male, 56 years); $\mathbf{d}$, $\mathbf{h}$ Coculture of $50 \%$ (P2) fat pad-derived adipose stem cells (ASC; male, 19 years) with $50 \%$ primary human meniscus cells (MCs; male, 56 years). Scale bar $100 \mu \mathrm{m}$

culture conditions. All constructs treated with secondary antibodies only proved negative for the type I and II collagen (data not shown).

Interestingly, SMG significantly downregulated the mRNA expression of SOX9 in pure MCs by 3.3-fold ( $p=0.02$; Fig. $5 d$ ). Similarly, SMG downregulated the expression of SOX9 in pure ASCs and in cocultures of $25 \% \mathrm{MC}$ and $75 \%$ ASC but not in coculture of $50 \%$ cells where a moderate increase of 1.2 -fold was observed. These modulations in SOX9 gene expression were not statistically significant with $p$-values $>0.05$.
SMG significantly enhanced the mRNA expression of the hypertrophic differentiation marker, COL10A1 (Fig. 5e). The increase was two-fold in pure MC $(p=0.008), 7.8$-fold in pure ASC $(p=0.03), 4.6$-fold in cocultures of $25 \%$ MC and $75 \%$ ASC ( $p=$ 0.0009 ), and 2.7 -fold in cocultures of $50 \% \mathrm{MC}$ and $50 \%$ ASC ( $p=$ $0.01)$. We evaluated the gene expression of established inhibitor of hypertrophic differentiation, gremlin 1 (GREM1), to provide some mechanistic insight into the noted SMG-mediated upregulation of COL1OA1 (Fig. 5f). SMG downregulated the mRNA level of GREM1 by two-fold $(p=0.02)$ in pure MC but not significantly in pure ASC (1.4-fold; $p=0.17$ ). SMG significantly downregulated GREM1 


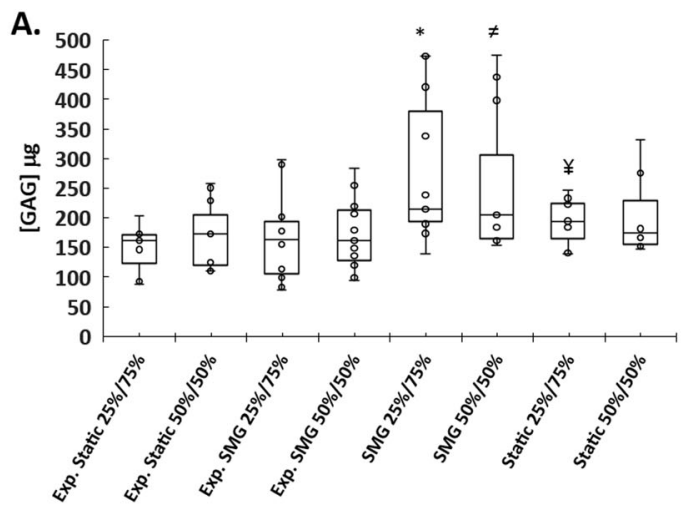

B.

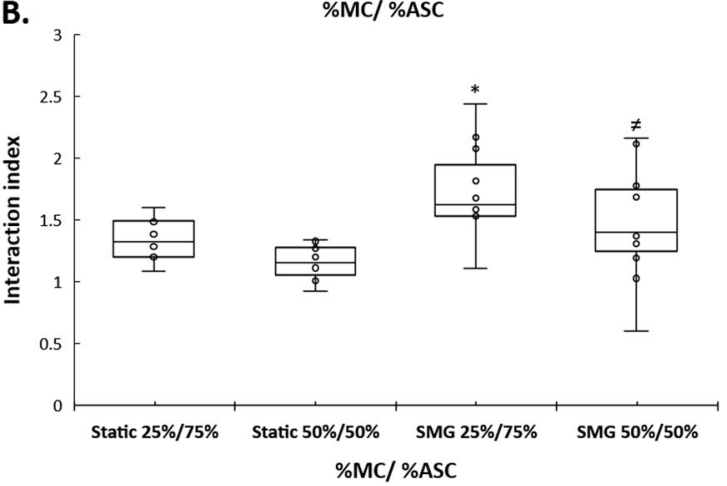

C.

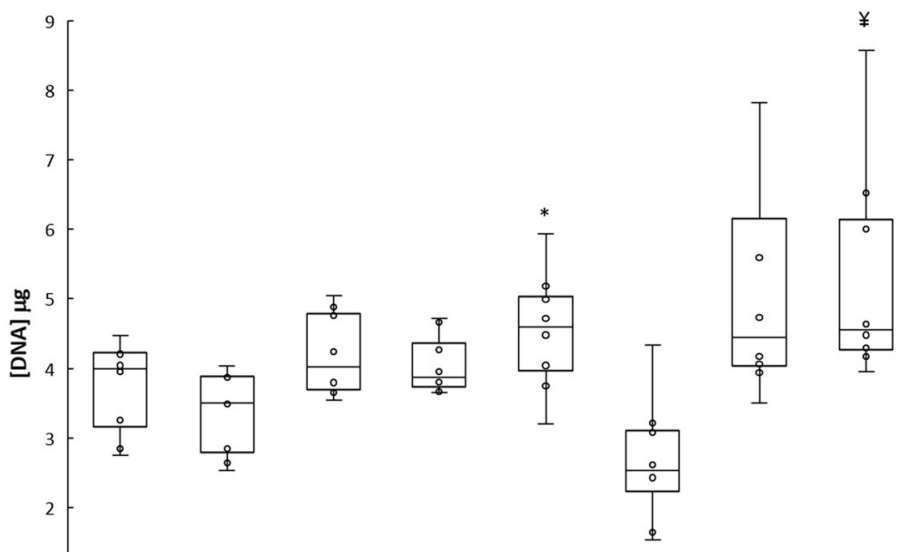

$\% \mathrm{MC} / \% \mathrm{ASC}$

Fig. 4 The interaction between primary human meniscus cells and fat pad-derived adipose stem cells on type I collagen scaffold after chondrogenic stimulation under static and simulate microgravity (SMG) conditions. Values are mean \pm SD of four independent experiments. Expected GAG values are indicated with an "Exp." prefix. The following symbols: * ${ }^{\neq}$and ${ }^{*}$ indicates statistically significant differences between expected and measured GAG values of cocultured group MC/ASC. a ${ }^{*}$ Exp. SMG $25 \% / 75 \%$ vs. SMG 25\%/75\%, $50 \% / 50 \%,{ }^{*}$ Exp. Static $25 \% / 75 \%$ vs. Static $25 \% / 75 \%$. b Interaction index values were determined from the ratio of measured and expected GAG as per the definition in section "Materials and methods". Symbols *and * indicate Wilcoxon signed rank test determined significant differences between the interaction indices of static and simulated microgravity (SMG) cocultured groups; ${ }^{*}$ SMG $25 \% / 75 \%$ vs. Static $25 \% / 75 \%$ and ${ }^{\neq}$SMG $50 \% / 50 \%$ vs. Static $50 \% / 50 \%$. c DNA contents of all groups after static and simulated microgravity culture conditions. Symbols ${ }^{*}$ and ${ }^{*}$ indicate a paired one-tailed Student's t-test (with unequal variance) statistically significant difference between the DNA contents of static and simulated microgravity (SMG) groups: ${ }^{*}$ SMG $100 \%$ MC vs. static $100 \%$ MC, and ${ }^{\ddagger}$ SMG $50 \% / 50 \%$ vs. static $50 \% / 50 \%$

expression by 3.9 -fold in cocultures of $25 \%$ MC and $75 \%$ ASC ( $p=$ $0.009)$, and by 12.4 -fold in $50 \%$ MC and $50 \%$ ASC cocultures $(p=$ 0.0001). Moreover, the mRNA expression of COL10A1 and GREM1 inversely correlated as assessed by Pearson correlation coefficient $(-0.166)$. The correlation was not statistically significant $(p=0.100$; one-tailed; Fig. 5g). In an analogous manner to collagen X, SMG increased the mRNA expression of MMP-13, another marker of hypertrophic differentiation of chondrocytes (Fig. 5h). It significantly increased the expression of MMP-13: in pure MC by five-fold $(p=0.0001)$, in pure ASC by five-fold $\left(p=1.78548 \times 10^{-6}\right), 3.7$-fold in cocultures of $25 \% \mathrm{MC}$ and $75 \%$ ASC $(p=0.0003)$, and 2.1 -fold in coculture of $50 \%$ MC and $50 \%$ ASC ( $p=0.008)$. Pearson correlation demonstrated a highly significant inverse relationship between the expression of GREM1 and MMP-13 $(r=-0.305 ; p=0.006$; onetailed; Fig. 5i).

We used immunofluorescence to confirm the translation of collagen $X$ in the constructs (Fig. 7). Static culture of pure MC appears to have the least deposition of collagen $X$ in the ECM (Fig. 7a). In contrast, the ECM of the pure MC after SMG culture appears to be more positive for type $X$ collagen epitope (Fig. 7e). Similarly, SMG culture of pure ASC (Fig. 7f) appears to be more positive for type $X$ collagen epitope than its static culture counterpart (Fig. 7b). Interestingly, in the cocultured constructs containing MC and ASC, the epitope of type $X$ collagen was strikingly evident around structures (indicated by white arrows) characterized by a hypertrophic phenotype due to their enlarged size and lacunaelike appearance (Fig. 7c, d, g and h). These structures appear to be larger after SMG culture (Fig. 7g) relative to static culture (Fig. $7 \mathrm{c}$ ), when MC and ASC cocultures are at a ratio of $25-75 \%$, respectively. However, it is interesting to note that the presence of type $X$ collagen epitope around the lacunae-like structures appear to be less evident in SMG cocultures of $50 \% \mathrm{MC}$ and $50 \%$ ASC, but more so in the extracellular space (Fig. 7h) compared to their static cocultured counterparts (Fig. 7d). All constructs treated with secondary antibodies only proved negative for the type I and Il collagen (data not shown).

\section{DISCUSSION}

The functional matrix of the knee meniscus is central to its biomechanical functionality within the joint. Generation of functional meniscus constructs, via tissue engineering and regenerative medicine strategies with a suitable cell source has the potential to revolutionize the treatment of avascular meniscus injuries, which do not heal. Avascular meniscus injuries account for most of meniscal lesions necessitating partial meniscectomies, which unfortunately predisposes the knee to early onset of osteoarthritis. Naturally, primary human MC are the optimal cell source for the generation of functional meniscal substitutes; however, limited availability from surgical specimen of partial meniscectomies is a drawback. Cell expansion strategies to increase numbers mitigate the expression of the functional matrix genes of the meniscus. ${ }^{18,19}$ To this end, direct coculture of primary human MC with adult-derived MSCs has been explored, ${ }^{20,21}$ to emulate the synergistic synthesis (i.e. chondro-induction) of the functional matrix of cartilage after coculture of primary articular or 
A.

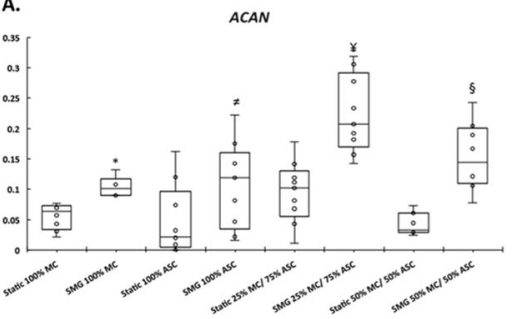

B.

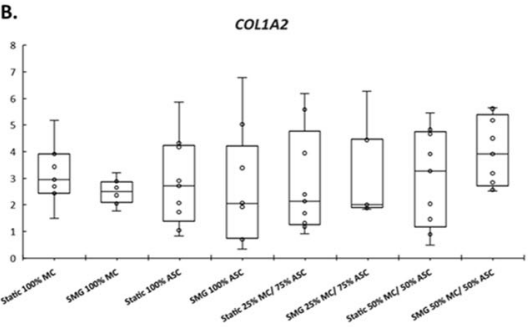

c. COLZAI

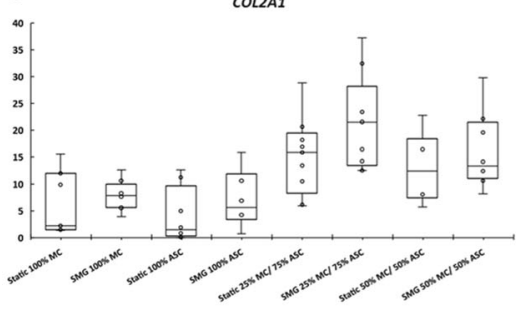

E.

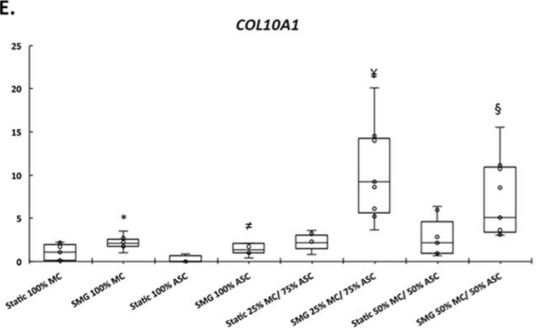

D.

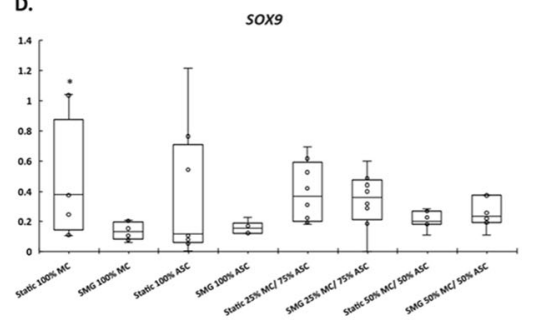

F.

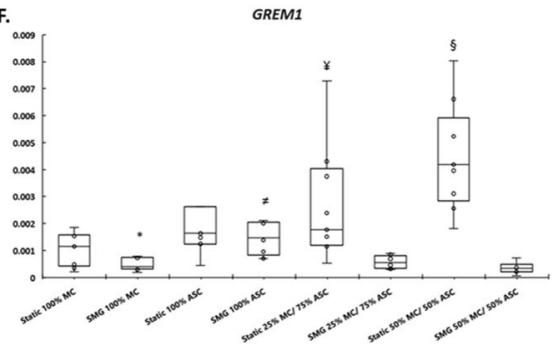

G.

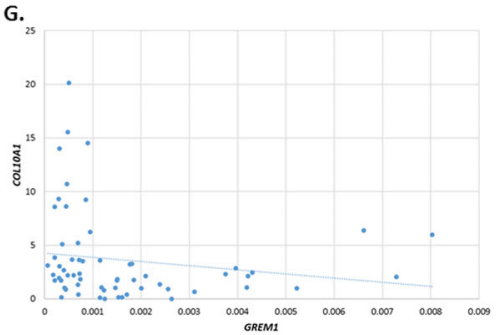

H.
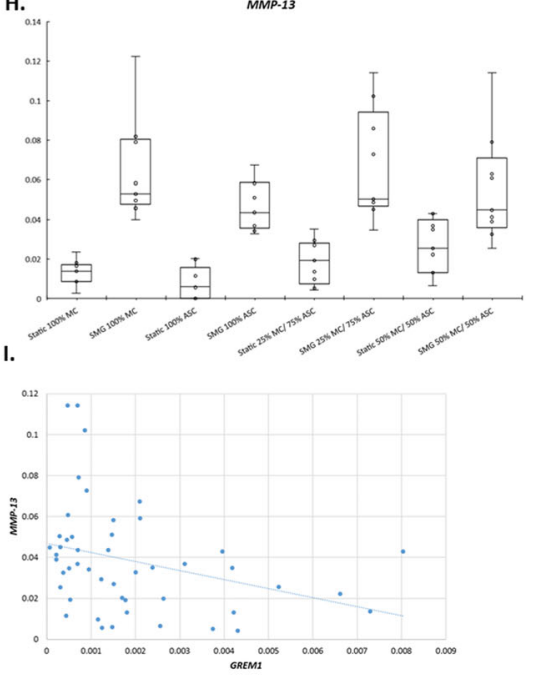

Fig. 5 Gene expression of primary human meniscus cells, fat pad-derived adipose mesenchymal stem cells and cocultured cells under static and simulated microgravity culture conditions. Symbols $*, \neq, ¥$ and ${ }^{\S}$ indicate a paired Student's $t$ test (one-tailed; unequal variance) statistically significant differences between the interaction indices of static and simulated microgravity (SMG) cocultured groups; ${ }^{*}$ SMG $100 \%$ MC vs. static $100 \%$ MC, ${ }^{\neq}$SMG $100 \%$ ASC vs. static $100 \%$ ASC, ${ }^{*}$ SMG $25 \% / 75 \%$ vs. static $25 \% / 75 \%$ and ${ }^{5}$ SMG $50 \% / 50 \%$ vs. static $50 \% / 50 \%$. a $A C A N$, b COL $1 A 2$, c COL2A1, d SOX9, e COL1OA1 and f GREM1. g Relative gene expression of COL10A1 was plotted against the relative gene expression of GREM1. Pearson correlation analysis was used to assess the relationship between COL10A1 and GREM1. h MMP-13. i Relative gene expression of MMP-13 was plotted against the relative gene expression of GREM1. Pearson correlation analysis was used to assess the relationship between MMP-13 and GREM1

nasal chondrocytes with bone marrow or adipose $\mathrm{MSCs}^{41}$ Simulated microgravity has been shown to synergistically enhance the synthesis of the functional matrix of articular cartilage in chondrocyte seeded polymeric scaffolds. ${ }^{35,42}$ Moreover, SMG synergistically enhanced the chondrogenic differentiation of adipose MSCs in the presence of TGF $\beta 1 .{ }^{30}$ These reported findings prompted us to ask the following question: can SMG further augment chondro-induction in direct cocultures of primary human MC and adipose MSCs? We hypothesized that SMG will further enhance chondro-induction in primary human MC and adipose MSCs in the presence of TGF $\beta$.

To investigate the hypothesis, human cells: adipose-derived MSCs and MC were successfully isolated from knee fat pad and menisci, respectively. Direct coculture of these cells in a 3D porous type I collagen matrix scaffolds, as a mimic of the predominant ECM of the native meniscus, was successfully demonstrated using differential fluorescent cell labeling. After 4 weeks of chondrogenic culture in the presence of TGF $\beta 3$, both pure cells and cocultured cells synthesized cartilaginous ECM under static and SMG.

Our study findings supported our hypothesis with evidence of GAG and ACAN increase. Moreover, SMG increased the DNA content of constructs containing pure MCs and cocultured MCs and ASCs. This was not the case in constructs containing pure ASCs. This finding suggested that the mechanism underlying SMG enhanced chondro-induction may involve increased proliferation of MC. Increased proliferation of chondrocytes in coculture with MSCs has also been reported previously as a mechanistic component of chondro-induction. ${ }^{41}$ An unexpected finding was the accompaniment of the microgravity enhanced chondroinduction with increased transcription of COL10A1 and MMP-13, markers for hypertrophic chondrocytes and hypertrophic differentiation of MSCs. ${ }^{43-48}$ Previous work had demonstrated that direct coculture of primary ${ }^{22}$ or early passage ${ }^{21}$ human MC with MSCs suppressed hypertrophic differentiation of MSCs. The mechanism underlying this unexpected finding appears to be associated with the downregulation of GREM1, a known inhibitor/ antagonist of hypertrophic differentiation. ${ }^{49}$ Parametric Pearson's correlation coefficient was used to determine the strength of the association between GREM1 and two hypertrophic markers: COL10A1 and MMP-13. Our assessment revealed an inverse correlation $(r=-0.166)$ between COL1OA1 and GREM1, albeit with a non-significant $p$ value. There was a highly significant inverse correlation $(r=-0.305, p=0.006)$ between the expression of MMP13 and GREM1. The potential of increased hypertrophic differentiation through GREM1 downregulation suggests a potential risk for enhanced bone formation in vivo through endochondral ossification pathway; conditional deletion of GREM1 caused a transient increase in bone formation and bone mass. ${ }^{50}$ With that in mind, it is unclear at this point if outer and inner MC will behave differently in their capacity to modulate the expression of GREM1 under SMG. Our previous work demonstrated that outer primary human $M C$ have a greater capacity to suppress COL1OA1 and MMP-13 expression. ${ }^{22}$ However, the study was conducted under static conditions using the cell pellet model of in vitro chondrogenesis. One may speculate that the bulk of primary human 

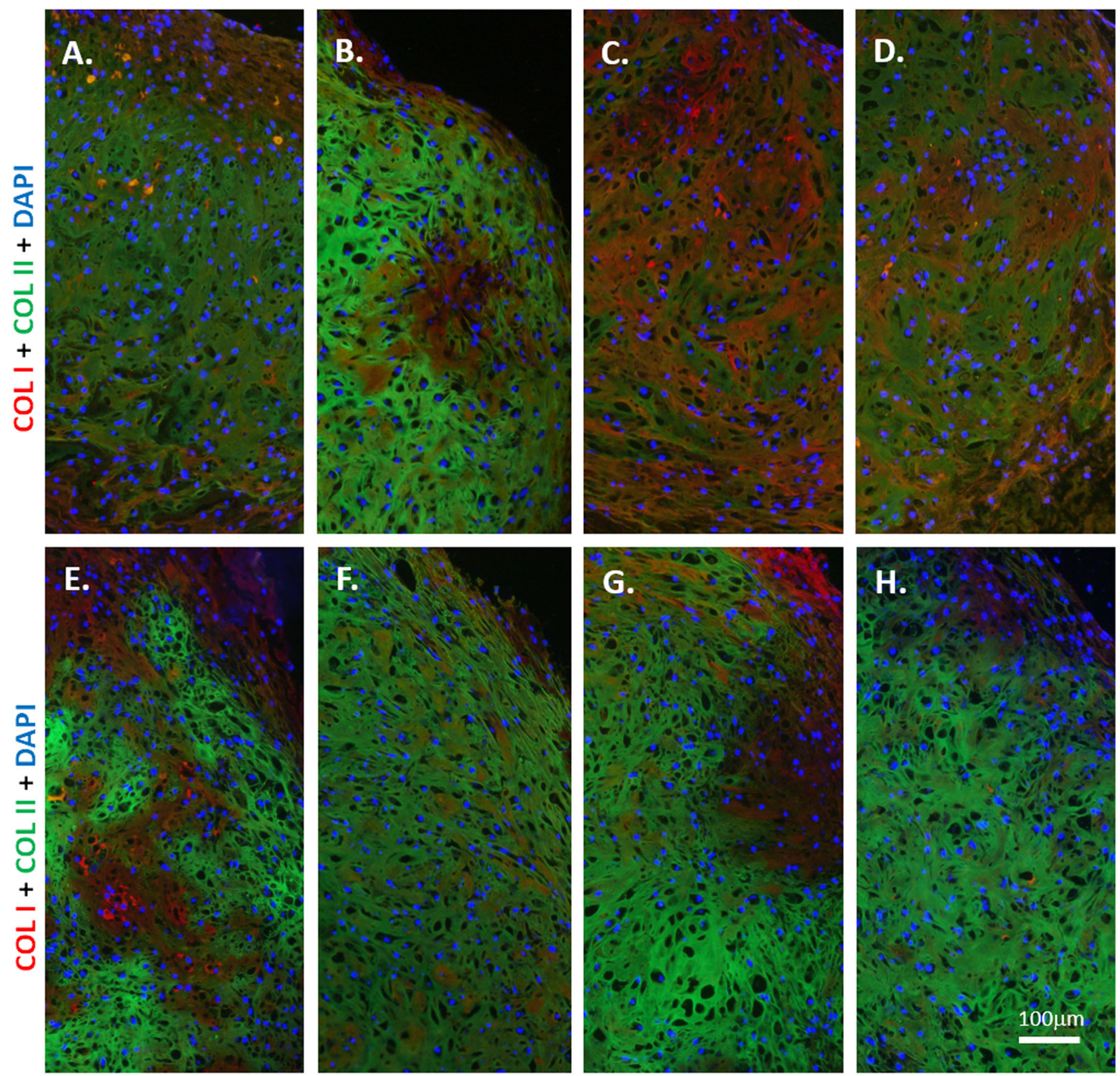

Fig. 6 Immunofluorescence of types I and II collagen after chondrogenic stimulation and differentiation of primary human meniscus cells and fat pad-derived adipose stem cells in porous type I collagen scaffolds. Representative immunofluorescence of cell-scaffold constructs after 4 weeks of culture in a defined serum-free chondrogenic medium containing transforming growth factor- $\beta 3$ (TGF $\beta 3$ ) and dexamethasone (DEX) under static $\mathbf{a}-\mathbf{d}$ and simulated microgravity $\mathbf{e}-\mathbf{h}$ conditions: $\mathbf{a}$, e pure primary human meniscus cells (MCs; male, 56 years); $\mathbf{b}, \mathbf{f}$ Passage 2 (P2) fat pad-derived adipose stem cells (ASC; male, 19 years); c, $\mathbf{g}$ coculture of $75 \%$ (P2) fat pad-derived adipose stem cells (ASC; male, 19 years) with $25 \%$ primary human meniscus cells (MCs; male, 56 years); $\mathbf{d}$, $\mathbf{h}$ Coculture of $50 \%$ (P2) fat pad-derived adipose stem cells (ASC; male, 19 years) with $50 \%$ primary human meniscus cells (MCs; male, 56 years). Scale bar $100 \mu \mathrm{m}$

$M C$ in this study may have originated from the inner region of the meniscus, since the inner portion of the tissue accounts for twothirds of the meniscus' width.

Our finding of increased COL10A1 expression in pure adiposederived MSCs under SMG is consistent with the reports of $\mathrm{Yu}$ et al. ${ }^{30}$ The authors demonstrated that SMG enhanced the in vitro chondrogenesis of adipose-derived MSCs in the presence of TGF $\beta 1$ but with increased COL1OA1 mRNA expression. They also demonstrated that SB203580, a highly specific and potent inhibitor of p38 mitogen-activated protein kinases (MAPK) signaling pathway, significantly suppressed the mRNA expression of COL10A1 during SMG culture conditions, suggesting that p38 MAPK was a significant player in the transduction of physical forces mediated by SMG. Thus, activation of p38 MAPK signaling may be an additional underlying mechanism contributing to the synergistic chondro-induction in our study under SMG. It is noteworthy that while Yu et al. ${ }^{30}$ reported an increase in COL2A1 and SOX9 under SMG, we did not observe a significant increase in the expression of these genes under SMG. But our findings on $A C A N$ and COL1OA1 transcriptional increase under SMG were consistent with the findings of $\mathrm{Yu}$ et al. ${ }^{30}$

In summary, the present study demonstrated that SMG enhanced the chondro-induction in cocultures of primary human $M C$ and adipose-derived mesenchymal stem but at the cost of increased hypertrophic differentiation that is concomitant with GREM1 downregulation. 

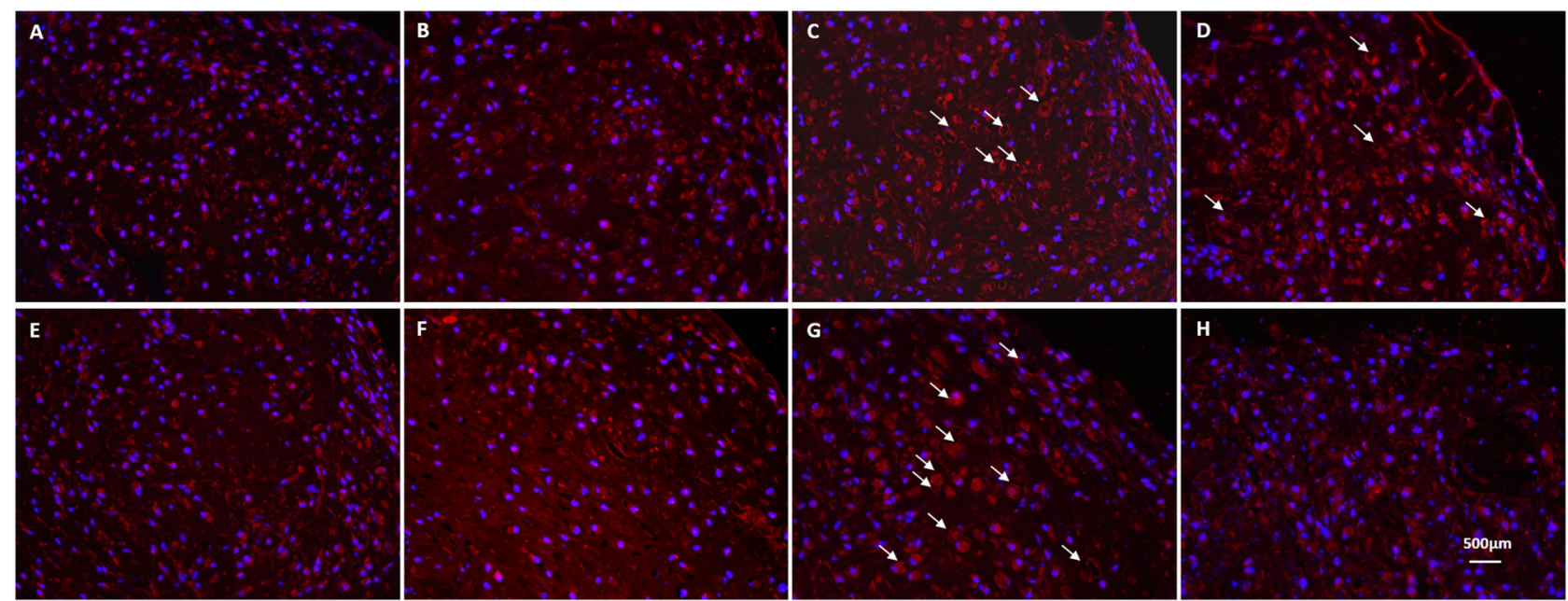

Fig. 7 Immunofluorescence of type $X$ collagen after chondrogenic stimulation and differentiation of primary human meniscus cells and fat pad-derived adipose stem cells in porous type I collagen scaffolds. Representative immunofluorescence of cell-scaffold constructs after 4 weeks of culture in a defined serum-free chondrogenic medium containing transforming growth factor- $\beta 3$ (TGF $\beta 3$ ) and dexamethasone (DEX) under static $\mathbf{a}-\mathbf{d}$ and simulated microgravity $\mathbf{e}-\mathbf{h}$ conditions: $\mathbf{a}$, e pure primary human meniscus cells (MCs; male, 56 years); $\mathbf{b}, \mathbf{f}$ Passage 2 (P2) fat pad-derived adipose stem cells (ASC; male, 19 years); c, $\mathbf{g}$ coculture of $75 \%$ (P2) fat pad-derived adipose stem cells (ASC; male, 19 years) with $25 \%$ primary human meniscus cells (MCs; male, 56 years); d, h coculture of $50 \%$ (P2) fat pad-derived adipose stem cells (ASC; male, 19 years) with $50 \%$ primary human meniscus cells (MCs; male, 56 years). Lacunae-like structures are indicated by white arrows. Scale bar $500 \mu \mathrm{m}$

\section{METHODS}

Ethics statement

Approval of the Health Research Ethics Board-Biomedical Panel of the University of Alberta, Edmonton, Canada was obtained for this study, and institutional safety and ethical guidelines were followed. Ethics committee waived the need for written informed consent of patients, as specimens used in the study were intended for discard in the normal course of the surgical procedure. Extensive precautions were taken to preserve the privacy of the participants donating specimens.

\section{Isolation of primary human MC}

Menisci were harvested from the knee joint of five donors: three males (14, 56, and 56) and two females (age 57 and 75), rinsed three times with sterile phosphate buffered saline (PBS), and finely minced. Donor information is documented in Table 2. MC were released from meniscus pieces by incubation for 16 hours at $37^{\circ} \mathrm{C}$ in type II collagenase $(0.15 \% \mathrm{w} / \mathrm{v}$ of 300 units/mg solid; Worthington, Lakewood, NJ, USA) in a standard medium, high glucose Dulbecco's modified Eagle's medium (DMEM) $4.5 \mathrm{mg} / \mathrm{ml}$ Dglucose supplemented with $5 \% \mathrm{v} / \mathrm{v}$ fetal bovine serum (FBS), 100 units $/ \mathrm{ml}$ penicillin and 100 units $/ \mathrm{ml}$ streptomycin, with L-glutamine $(2 \mathrm{mM})$ (Invitrogen, Mississauga, Ontario, Canada), then filtered through a 150$\mu \mathrm{m}$ nylon mesh to remove cellular debris, as described previously. Isolated cells were plated in standard medium supplemented in $150 \mathrm{~cm}^{2}$ tissue culture plastic (TCP) at $37{ }^{\circ} \mathrm{C}$ and $21 \% \mathrm{O}_{2}$ in a humidified incubator with $5 \%$ $\mathrm{CO}_{2}$. After $48 \mathrm{~h}$ of recovery in standard medium, the cells were detached with $0.15 \% \mathrm{w} / \mathrm{v}$ trypsin-EDTA in Hank's buffered saline solution (HBSS; Invitrogen) and counted prior to use.

\section{Isolation of human adipose stem cells}

Patellar fat pads were harvested from the knee joint of five donors undergoing routine orthopedic procedure; three males (age 17, 19 and 40) and two females (age 20 and 21). Donor information is documented in Table 2. The adipose tissue was rinsed three times with PBS, and finely minced before enzymatic digestion. Tissue was digested with type II collagenase $\left(0.15 \% \mathrm{w} / \mathrm{v}\right.$ of 300 units $/ \mathrm{mg}$ solid; Worthington) in PBS at $37^{\circ} \mathrm{C}$ using a water bath shaker at $150 \mathrm{rpm}$ for 45 minutes. Nucleated cells were re-suspended in alpha modified Eagle's medium (aMEM) supplemented with $10 \% \mathrm{v} / \mathrm{v} \mathrm{FBS}$, and $1 \% \mathrm{w} / \mathrm{v}$ antibiotic/antimycotic solution filtered through a $100 \mu \mathrm{m}$ nylon mesh to remove cellular debris. The number of nucleated cells in the aspirates was determined by crystal violet nuclei staining with cell counting using a hemocytometer. Thereafter, 15 million mono-nucleated cells (MNCs) were seeded per $150 \mathrm{~cm}^{2}$ TCP. The culture medium was aMEM supplemented with $10 \% \mathrm{v} / \mathrm{v} \mathrm{FBS}$, penicillin-streptomycin, 4-(2-hydroxyethyl)-1- piperazineethanesulfonic acid (HEPES), sodium pyruvate (all from Invitrogen) and $5 \mathrm{ng} / \mathrm{ml}$ basic fibroblast growth factor (bFGF or FGF2; from Humanzyme, Medicorp Inc., Montreal, Quebec, Canada) to maintain multipotency. ${ }^{51-54}$ The cells were cultured until passage $2(\mathrm{P} 2)$ at $37^{\circ} \mathrm{C}$ under $95 \%$ humidity in an atmosphere of $21 \% \mathrm{O}_{2}$ and $5 \% \mathrm{CO}_{2}$.

\section{Colony-forming unit fibroblastic (CFU-F) assay}

A CFU-F assay was performed to determine the proportion of plasticadherent cells from adipose-derived MNCs. MNCs from each donor were plated in triplicates at 500 MNCs per $100 \mathrm{~mm}$ diameter sterile Petri dish (Becton Dickinson Canada Inc.) and cultured for expansion as described above at $37{ }^{\circ} \mathrm{C}$ under $95 \%$ humidity in an atmosphere of $21 \% \mathrm{O}_{2}$ and $5 \%$ $\mathrm{CO}_{2}$. After the first week, the non-adherent cell population was removed by aspiration and culture media was replenished twice for another week. After the 2 weeks of culture, the culture media was aspirated and the Petri dish was washed with sterile PBS, then fixed with $10 \% \mathrm{w} / \mathrm{v}$ buffered formalin for 5 minutes. The formalin was aspirated and the Petri dish was washed twice with PBS, and stained with $0.25 \% \mathrm{w} / \mathrm{v}$ crystal violet solution (Sigma-Aldrich). Stained colonies were revealed after washing with a copious amount of distilled water. Each stained cell collection was assessed and considered to be a colony as previously described. ${ }^{55}$ The number of colonies developed from the total number of MNC seeded was used to calculate clonogenicity (\%), with each colony representing a single cellderived clone.

\section{Flow cytometry analysis}

All primary monoclonal antibodies used herein were directly conjugated antibodies to fluorescein isothiocynate (mAb-FITC) or to phycoerythrin (mAbPE). Antibodies were either from BD Pharmingen or Invitrogen-see Table 1. The cells were analyzed on a FACScan flow cytometer (Becton Dickinson) after detachment from culture flask by $0.05 \% \mathrm{w} / \mathrm{v}$ trypsin-EDTA (Invitrogen). Staining buffer was prepared with PBS containing $1 \% \mathrm{w} / \mathrm{v}$ BSA (Sigma). The cells were re-suspended in $4^{\circ} \mathrm{C}$ cold staining buffer at $5 \times 10^{6} / \mathrm{ml}$. Thereafter, the cells were dispensed into sample tubes $(12 \times 75 \mathrm{~mm}$ polystyrene roundbottom tubes, Becton Dickinson) in $20 \mu \mathrm{l}$ aliquots and incubated for 15 minutes with the antibodies at $4{ }^{\circ} \mathrm{C}$. All incubations were implemented in $5 \mathrm{ml}$ dilution tubes at room temperature in the dark and all washing steps were performed by a combination of centrifugation ( $400 \mathrm{~g}, 5$ minutes) and aspiration of supernatant. Staining buffer $(200 \mu \mathrm{l})$ was then added to the tubes and cells were incubated for 10 additional minutes. After removal of the supernatant by centrifugation, cells were washed with PBS and kept cold before analysis by flow cytometry. Non-specific staining was assessed using relevant isotype controls. Single color immuno-fluorescence analysis for the different surface markers was performed with mAb-FITC and mAb-PE. Data 
Table 1. Antibodies used to characterize infrapatellar fat pad mesenchymal stem cells

\begin{tabular}{|c|c|c|c|}
\hline Specificity & Isotype* & Cat.\#/Flurochrome & Source \\
\hline CD29 & mlgG1 & CD2901/FITC & Invitrogen \\
\hline CD44 (Pgp-1, H-CAM, Ly 24) & $\operatorname{mlgG} 2 \mathrm{~b}$ & 560977/FITC & BD Pharmingen \\
\hline CD45 (2B11) & $\operatorname{mlgG} 1$ & Sc-20056/PE & Santa Cruz Biotechnology \\
\hline CD105 (endoglin) & mlgG1 & sc-71043/PE & Santa Cruz Biotechnology \\
\hline CD151 (PETA-3) & mlgG1 & $556057 / \mathrm{PE}$ & BD Pharmingen \\
\hline CD184 (CXCR4) & $\operatorname{mlgG} 2 a$ & 560937/PE-СуTM5 & BD Pharmingen \\
\hline Not specified (Isotype control) & $\operatorname{mlgG1}$ & sC-2855/FITC & Santa Cruz Biotechnology \\
\hline Not specified (Isotype control) & mlgG1 & sc-2866/PE & Santa Cruz Biotechnology \\
\hline
\end{tabular}

Table 2. Donor information of meniscal (MEN/ NMEN) and patellar fat pad (ASC) specimen

\begin{tabular}{lllllll}
\hline Donor & Age (years) & Gender (M/F) & Weight $(\mathrm{kg})$ & Height $(\mathrm{cm})$ & Smoker? (Y/N) & Medical history/surgery reason \\
\hline MEN155 & 56 & M & 140 & 186 & $\mathrm{~N}$ & Osteoarthritis \\
MEN159 & 75 & $\mathrm{~F}$ & 93 & 155 & $\mathrm{~N}$ & No anticancer drug use/osteoarthritis \\
MEN161 & 56 & $\mathrm{M}$ & 66 & 168 & $\mathrm{Y}$ & No anticancer drug use/osteoarthritis \\
MEN172 & 57 & $\mathrm{~F}$ & 96 & 151 & $\mathrm{~N}$ & Osteoarthritis \\
NMEN190 & 14 & $\mathrm{M}$ & 65 & 175 & $\mathrm{~N}$ & Partial meniscectomy \\
ASC64 & 20 & $\mathrm{~F}$ & - & - & - & Mitral valve prolapse/knee injury \\
ASC68 & 19 & $\mathrm{M}$ & 69.5 & 173 & - & Healthy/meniscal \& ACL repair \\
ASC67 & 17 & $\mathrm{M}$ & 79.5 & 185 & $\mathrm{~N}$ & Knee joint injury \\
ASC70 & 21 & $\mathrm{~F}$ & 71 & 161 & $\mathrm{Y}$ & Allergy to nickel, asthma \\
ASC97 & 40 & $\mathrm{M}$ & 88.9 & 180 & $\mathrm{Y}$ & Knee repair \\
\end{tabular}

acquisition was performed with Cellquest software (Becton Dickinson). FITC emission was measured at FL1 and PE at FL3. For each sample a region for live cells was defined, according to the Forward Scatter (FSC) and Side Scatter (SSC) signals, which excluded aggregated cells from the analysis. Data analysis was performed with Cyflogic version 1.2.1, Perthu Terho \& CyFlo Ltd., Finland. For each surface marker analyzed, percentage of positive cells and the level of marker expression were calculated. Percentage of positive cells was calculated as the percentage of cells having a measured fluorescence greater than that of $99.5 \%$ of the cells stained with each associated isotype control. Cells were considered positive for a surface marker when the percentage of positive cells for that surface marker was $\geq 6 \%$. The level of expression of each marker was calculated as the ratio between geometric mean fluorescence intensity (MFI) of samples and that of the isotype control.

Type I collagen scaffold constructs

Type I collagen matrix (Integra Lifesciences, PlainsBoro, NJ, USA; $10 \mathrm{~cm} \times$ $12.5 \mathrm{~cm} ; 3.5 \mathrm{~mm}$ total thickness collagen sponge with pore size of $115 \pm 20$ $\mu \mathrm{m}$ ) was cut into $6 \mathrm{~mm}$ diameter disks using a sterile biopsy punch. To limit variability between batches of scaffolds, disks taken from the same batch of scaffolds were used for each experiment. The disks were placed in a 24well plate for seeding with cells.

\section{Co-culture of MCs and ASCs}

Cells were co-cultured in $25 \%: 75 \%$ and $50 \%: 50 \%$ ratios of MCs to ASCs, in addition to controls of each cell type alone. MCs and ASCs were sex matched, but obtained from different donors. After counting, cells were combined in the required ratios, and were carefully seeded on the Type I collagen scaffold via a micropipette with a total of 250,000 cells suspended in $20 \mu \mathrm{l}$ of a defined serum-free chondrogenic medium consisting of high glucose DMEM containing $0.1 \mathrm{mM}$ non-essential amino acids, $1 \mathrm{mM}$ sodium pyruvate, $100 \mathrm{mM}$ HEPES buffer, $1 \mathrm{mM}$ sodium pyruvate, $100 \mathrm{U} /$ $\mathrm{ml}$ penicillin, $100 \mathrm{mg} / \mathrm{ml}$ streptomycin, $0.29 \mathrm{mg} / \mathrm{ml}$ L-glutamine (Invitrogen) supplemented with $0.1 \mathrm{mM}$ ascorbic acid 2-phosphate, $40 \mathrm{mg} / \mathrm{ml}$ L-proline, $10^{-7} \mathrm{M}$ dexamethasone, $1 \times \mathrm{ITS}+1$ premix (Sigma-Aldrich, Oakville, Canada), $10 \mathrm{ng} / \mathrm{ml}$ TGF- $\beta 3$ (Humanzyme-Medicorp Inc.). The seeded disks were transferred to a humidified incubator at $37^{\circ} \mathrm{C}$ with $21 \% \mathrm{O}_{2}$ and $5 \%$ $\mathrm{CO}_{2}$ for 15 minutes to allow initial cell attachment. Thereafter, $100 \mu \mathrm{l}$ of chondrogenic medium was gently added to the base of each well containing cell-seeded disks followed by a 30 minutes incubation period in a humidified incubator at $37{ }^{\circ} \mathrm{C}$ with $21 \% \mathrm{O}_{2}$ and $5 \% \mathrm{CO}_{2}$. After the incubation period, $700 \mu \mathrm{l}$ of chondrogenic medium was added slowly to the base of each well until the entire seeded scaffolds were covered. The seeded scaffolds were then incubated at $37{ }^{\circ} \mathrm{C}$ with $21 \% \mathrm{O}_{2}$ and $5 \% \mathrm{CO}_{2}$ for 7 days. Chondrogenic media exchange was performed twice per week.

\section{Co-culture of fluorescently labeled MCs and ASCs}

To demonstrate co-culture of MCs and ASCs, isolated MCs and ASCs were labeled with fluorescent dyes in suspension according to manufacturer's labeling protocol. Pure MCs were labeled with PKH67 (green) and pure ASCs were labeled with PKH26 (red). The labeled cells were visualized in monolayer after plating in a T25 TCP flask using an Eclipse Ti-S microscope (Nikon Canada Mississauga, Canada) fitted with NIS Elements (version 4.20; Nikon Canada). PKH67 and PKH26 fluorescent cell linker kits were purchased from Sigma-Aldrich (Oakville, Canada). The labeled cells were seeded onto type I collagen matrix scaffolds as described above. Culture media, conditions, and duration were as described above but only static culture 
was implemented to demonstrate co-culture of labeled cells. Cells were mixed at a ratio of $25 \% \mathrm{MC}$ and $75 \%$ ASC prior to seeding on type I collagen matrix scaffold. Cell-scaffold constructs were cultured in chondrogenic medium as before for 28 days. Thereafter, the cultured constructs were embedded in a VWR clear frozen section compound (VWR, Mississauga, ON, Canada). Engineered tissue sections were cut on a Leica Biosystems cryostat at $7 \mu \mathrm{m}$. Images were captured as before and assembled in Image $J$ version $1.51 \mathrm{k}$ software $(\mathrm{NIH}$, Bethesda, Maryland, USA).

\section{Culture in SMG}

After 7 days of culture, one set of cell co-culture on type I collagen scaffolds (MC alone, ASC alone, 1:3 MC to ASC, and 1:1 MC to ASC) were transferred to $10 \mathrm{~mL}$ vessels and inserted into a rotary bioreactor RCCS (Synthecon, Inc., Houston, TX). An identical set of co-cultures was used as a control, and continued to be incubated under static conditions. The rotary bioreactor was housed within the same incubator as the static controls, and both continued to be cultured in a humidified incubator at $37^{\circ} \mathrm{C}$ with $21 \% \mathrm{O}_{2}$ and $5 \% \mathrm{CO}_{2}$. The rotation speed of the RCCS increased from 20 to $30 \mathrm{rpm}$ over the course of 21 days of culture. This increase in rotation speed was required to keep the increasing mass of the scaffolds suspended under microgravity conditions. After 21 days of culture in the rotary bioreactor ( 28 days total from seeding of the scaffolds), the culture scaffolds were harvested and analyzed. Scaffolds were processed biochemically for GAG and DNA content, histologically and immunofluorescence for cartilage-specific matrix proteins, and at the molecular level by real time $\mathrm{qPCR}$ for gene expression analysis.

\section{Biochemical analysis}

After culture, scaffolds were rinsed in PBS (Invitrogen), and frozen at $-80^{\circ}$ C. For analysis, samples were digested in proteinase $\mathrm{K}(1 \mathrm{mg} / \mathrm{ml}$ in $50 \mathrm{mM}$ Tris with $1 \mathrm{mM}$ EDTA, $1 \mathrm{mM}$ iodoacetamide and $10 \mathrm{mg} / \mathrm{ml}$ pepstatin A-all from Sigma-Aldrich) for $16 \mathrm{~h}$ at $56^{\circ} \mathrm{C}$. The sulphated GAG content was measured by 1,9 dimethylmethylene blue (DMB) binding (Sigma-Aldrich) using chondroitin sulfate (Sigma-Aldrich) as standard. The DNA content was determined using the CyQuant cell proliferation assay kit (Invitrogen) with supplied bacteriophage $\lambda$ DNA as standard. Chondro-induction is defined as previously described.$^{41}$ Based on experimentally measured GAG contents of pure $\mathrm{MC}$ seeded scaffolds $\left(\mathrm{GAG}_{100 \%} \mathrm{MCs}\right)$ and pure ASC seeded scaffolds $\left(\mathrm{GAG}_{100 \%} \mathrm{Asc}\right)$, the expected total $\mathrm{GAG}$ ( $\left.\mathrm{GAG}_{\text {expected }}\right)$ in the coculture cell-seeded scaffolds was calculated as a linear function of the proportion (\%) of MC using the following equation:

$G A G_{\text {expected }}=\mathrm{GAG}_{100 \% \mathrm{MC}}+\left(\mathrm{GAG}_{100 \% \mathrm{MC}}-\mathrm{GAG}_{100 \% \mathrm{ASC}}\right) \times \% \mathrm{MC}$

The interaction index was then calculated as the ratio of the GAG measured in the cocultured cell-seeded scaffolds (GAG measured) to the GAG expected. When the interaction index is higher than 1, then chondroinduction is considered to have occurred. ${ }^{20,41}$

\section{Histology and immuno-histochemistry}

Tissues generated from the scaffold-cell cultures were fixed in $4 \% \mathrm{v} / \mathrm{v}$ phosphate buffered formalin, processed into paraffin wax, sectioned at 5 $\mu \mathrm{m}$ and stained with safranin $\mathrm{O}$ and counterstained with fast green, to reveal sulfated proteoglycan (GAG) matrix depositions. Other sections were probed with antibodies raised against collagen types I and II. The distribution of types I and II collagen were evaluated using immunofluorescence imaging. Sections were deparaffinized and rehydrated. Antigen retrieval was conducted with Protease XXV (Thermo Scientific) for 30 minutes at room temperature and hyaluronidase (H6254, Sigma) for $30 \mathrm{~min}$ at $37^{\circ} \mathrm{C}$. Sections were then blocked with $5 \% \mathrm{w} / \mathrm{v}$ bovine serum albumin in PBS for 30 minutes at room temperature. Collagen I, II and X labeling was completed using rabbit anti-collagen I, 1:200 dilution (CL50111AP-1, Cedarlane Labs, Burlington, ON, Canada), mouse anti-collagen II, 1:200 dilution (II-II6B3, Developmental Studies Hybridoma Bank, University of lowa, USA) and rabbit anti-collagen X (ab58632, Abcam, UK) using a 1:100 dilution, respectively, and incubated overnight at $4{ }^{\circ} \mathrm{C}$. Secondary labeling using goat anti-mouse IgG, Alexa Fluor 488, 1:200 (ab150117, Abcam) and goat anti-rabbit lgG, Alexa Fluor 594, 1:200 (ab150080, Abcam) incubated at room temperature for 1 hour. Slides were mounted using Everbrite Hardset Mounting Medium with DAPI (Biotium) and imaged using a Nikon Ti-S Microscope fitted with DS-U3/Fi2 Color CCD camera using FITC and Texas Red filters (Nikon Canada Mississauga, Canada) with NIS Elements software (version 4.20; Nikon Canada) and assembled in Image J version $1.51 \mathrm{k}$ software (NIH, Bethesda, Maryland, USA).
Gene expression analysis

Total RNA was extracted from MCs, ASCs, and scaffold cultures using Trizol (Invitrogen) after grinding with Molecular Grinding Resin (Geno Technology Inc., St Louis, USA) in combination with the use of RNeasy mini-kit (Qiagen, Mississauga, ON, Canada) and after removal of contaminating genomic DNA from the preparations with DNase treatment. Total RNA $(100 \mathrm{ng})$ in a $40 \mu \mathrm{l}$ reaction was reverse transcribed to cDNA using GoScript reverse transcriptase (Fisher Scientific, Whitby, Ontario, Canada) primed with random primer oligonucleotides. QPCR was performed either in a DNA Engine OpticonIContinuous Fluorescence Detection System (Bio-Rad) using hot start Taq and SYBR Green detection (Eurogentec North America Inc, San Diego, CA, USA) or in a Bio-Rad CFX connect real-time system (Bio-Rad Laboratories, Mississauga, ON, Canada). Primer sequences were either designed by Primer Express 3.0.1 (Applied Biosystems, ThermoFisher, ON, Canada) or taken from previously published work. All primers were obtained from Invitrogen, Mississauga, ON, Canada. Gene (mRNA) expression levels for each primer set were normalized to the expression level of human $\beta$ actin, by the $2-\Delta \Delta c t$ method. Primer sequence are as follows: $A C A N^{19}$ (Accession\# M55172) forward: AGG GCG AGT GGA ATG ATG TT; $A C A N^{19}$ reverse: GGT GGC TGT GCC CTT TाT AC; $A C T B^{19}$ (Accession\# NM_001101) forward AAG CCA CCC CAC TTC TCT CTA A; ACTB $^{19}$ reverse AAT GCT ATC ACC TCC CCT GTG T; COL1A2 ${ }^{19}$ (NM_000089) forward: TTG CCC AAA GTT GTC CTC TTC T; COL1A2 ${ }^{19}$ reverse: AGC TTC TGT GGA ACC ATG GAA; COL2A $1^{19}$ (Accession\# NM_033150) forward CTG CAA AAT AAA ATC TCG GTG TTC T; COL2A1 reverse: GGG CAT TTG ACT CAC ACC AGT; COL10A1 (Accession\# X60382) forward: GAA GTT ATA ATT TAC ACT GAG GGT TTC AAA; COL10A 1 reverse: GAG GCA CAG CTT AAA AGT TाT AAA CA; GREM-1 (Accession\# NM_001191322.1) forward: CAT GTG ACG GAG CGC AAA TA; GREM-1 reverse: GCT TAA GCG GCT GGG TाT T; MMP13 (Accession\# NM_002427) forward: CATCCAAAAACGCCAGACAA; MMP13 reverse: CGGAGACTGGTAATGGCATCA; SOX9 ${ }^{19}$ (Accession\# Z46629) forward: CTT TGG TIT GTG TTC GTG TTT TG; $S O X 9^{19}$ reverse: AGA GAA AGA AAA AGG GAA AGG TAA GTT T.

\section{Statistical analysis}

A total of five independent experiments were performed with five donor specimens for each cell type. Unless stated otherwise, numerical data distribution represents data from five donors and is presented as a boxplot of the minimum, first quartile, median, third quartile, and maximum values. Statistical analyses were performed using SPSS (version 23; IBM Canada Ltd, ON, Canada). Outliers were defined as values 1.5 interquartile ranges below the first quartile or above the third quartile. Data were assessed for normality using the Shapiro-Wilk test. Levene's test was used to assess homogeneity of error variances. Statistical test choice was dependent on outcome of normality and homogeneity test. Paired comparisons between static and SMG groups was analyzed using either Wilcoxon signed rank test or paired Student's $t$ test depending on outcome of normality test. Pearson or Spearman correlation coefficient was used to assess correlation between genes. Statistical significance was considered when $p<0.05$.

Data availability

All relevant data are available from the corresponding author.

\section{ACKNOWLEDGEMENTS}

Financial support was provided by Canadian Institutes for Health Research (CIHR MOP 125921), Canadian Foundation for Innovation (CFI 33786), University of Alberta Hospital Foundation (RES0028185) and Edmonton Orthopedic Research Committee (GS22000020). In-kind donation of type I collagen scaffolds was provided by Integra LifeSciences Inc., Plainsboro, NJ, USA.

\section{AUTHOR CONTRIBUTIONS}

W.M.W. was involved in study design, performed cell culture, experimental assays, data acquisition, and initial manuscript writing. A.M.S. performed cell isolation, cell culture, experimental assays and data acquisition. M.K. performed gene expression assays and associated data analysis. N.M.J. was responsible for specimen procurement and was involved in manuscript writing. A.B.A. conceived the study, supervised the study, performed data and statistical analysis and was responsible for writing of the final manuscript. All authors read and approved the final manuscript. 


\section{ADDITIONAL INFORMATION}

Competing interests: The authors declare no competing financial interests.

Publisher's note: Springer Nature remains neutral with regard to jurisdictional claims in published maps and institutional affiliations.

\section{REFERENCES}

1. Makris, E. A., Hadidi, P. \& Athanasiou, K. A. The knee meniscus: structure-function, pathophysiology, current repair techniques, and prospects for regeneration. Biomaterials 32, 7411-7431 (2011).

2. Andrews, S. H., Adesida, A. B., Abusara, Z. \& Shrive, N. G. Current concepts on structure-function relationships in the menisci. Connect. Tissue Res.. https://doi. org/10.1080/03008207.2017.1303489 (2017).

3. Roos, H. et al. Knee osteoarthritis after meniscectomy: Prevalence of radiographic changes after twenty-one years, compared with matched controls. Arthritis Rheum. 41, 687-693 (1998).

4. McDermott, I. D. \& Amis, A. A. The consequences of meniscectomy. J. Bone Jt. Surg. Br. 88, 1549-1556 (2006).

5. Sweigart, M. A. \& Athanasiou, K. A. Toward tissue engineering of the knee meniscus. Tissue Eng. JID - 9505538 7, 111-129 (2001).

6. Buma, P., Ramrattan, N. N., van Tienen, T. G. \& Veth, R. P. Tissue engineering of the meniscus. Biomaterials 25, 1523-1532 (2004).

7. Adams, S. B. Jr, Randolph, M. A. \& Gill, T. J. Tissue engineering for meniscus repair. J. Knee Surg. 18, 25-30 (2005).

8. Chiari, C. et al. A tissue engineering approach to meniscus regeneration in a sheep model. Osteoarthritis Cartil. 14, 1056-1065 (2006).

9. Kon, E. et al. Tissue engineering for total meniscal substitution: animal study in sheep model. Tissue Eng. Part A 14, 1067-1080 (2008).

10. Stapleton, T. W. et al. Development and characterization of an acellular porcine medial meniscus for use in tissue engineering. Tissue Eng. Part A 14, 505-518 (2008).

11. Baker, B. M., Nathan, A. S., Huffman, G. R. \& Mauck, R. L. Tissue engineering with meniscus cells derived from surgical debris. Osteoarthritis Cartil. 17, 336-345 (2009).

12. Zellner, J. et al. Role of mesenchymal stem cells in tissue engineering of meniscus. J. Biomed. Mater. Res. A 94, 1150-1161 (2010).

13. Kon, E. et al. Tissue engineering for total meniscal substitution: animal study in sheep model-results at 12 months. Tissue Eng. Part A 18, 1573-1582 (2012).

14. Cucchiarini, M. et al. Advances in combining gene therapy with cell and tissue engineering-based approaches to enhance healing of the meniscus. Osteoarthritis Cartil. 24, 1330-1339 (2016).

15. W. Niu, et al. Cell-based strategies for meniscus tissue engineering. Stem Cells Int. 2016, 4717184 (2016).

16. Adams, M. E., Hukins, D. W. L. in Knee Meniscus: Basic and Clinical Foundations (eds Mow, V. C., Arnoczky, S. P., Jackson, D. W.) 15-28 (Raven Press Ltd, New York, 1992).

17. Sanchez-Adams, J. \& Athanasiou, K. A. Biomechanics of meniscus cells: regional variation and comparison to articular chondrocytes and ligament cells. Biomech. Model. Mechanobiol. 11, 1047-1056 (2012).

18. Nakata, K. et al. Human meniscus cell: characterization of the primary culture and use for tissue engineering. Clin. Orthop. Relat. Res. 391, S208-S218 (2001).

19. Adesida, A. B., Grady, L. M., Khan, W. S. \& Hardingham, T. E. The matrix-forming phenotype of cultured human meniscus cells is enhanced after culture with fibroblast growth factor 2 and is further stimulated by hypoxia. Arthritis Res. Ther. 8, R61 (2006).

20. Matthies, N. F., Mulet-Sierra, A., Jomha, N. M. \& Adesida, A. B. Matrix formation is enhanced in co-cultures of human meniscus cells with bone marrow stromal cells. J. Tissue Eng. Regen. Med. 7, 965-973 (2013).

21. Cui, X., Hasegawa, A., Lotz, M. \& D'Lima, D. Structured three-dimensional coculture of mesenchymal stem cells with meniscus cells promotes meniscal phenotype without hypertrophy. Biotechnol. Bioeng. 109, 2369-2380 (2012).

22. Saliken, D. J., Mulet-Sierra, A., Jomha, N. M. \& Adesida, A. B. Decreased hypertrophic differentiation accompanies enhanced matrix formation in co-cultures of outer meniscus cells with bone marrow mesenchymal stromal cells. Arthritis Res. Ther. 14, R153 (2012).

23. Aung, A., Gupta, G., Majid, G. \& Varghese, S. Osteoarthritic chondrocyte-secreted morphogens induce chondrogenic differentiation of human mesenschymal stem cells. Arthritis Rheum. 63, 148-158 (2011).

24. Johnstone, B., Hering, T. M., Caplan, A. I., Goldberg, V. M. \& Yoo, J. U. In vitro chondrogenesis of bone marrow-derived mesenchymal progenitor cells. Exp. Cell Res. 238, 265-272 (1998).

25. Adesida, A. B., Mulet-Sierra, A. \& Jomha, N. M. Hypoxia mediated isolation and expansion enhances the chondrogenic capacity of bone marrow mesenchymal stromal cells. Stem Cell Res. Ther. 3, 9 (2012).
26. Li, Z., Kupcsik, L., Yao, S. J., Alini, M. \& Stoddart, M. J. Mechanical load modulates chondrogenesis of human mesenchymal stem cells through the TGF-beta pathway. J. Cell. Mol. Med. 14, 1338-1346 (2010).

27. Park, S. R., Choi, B. H. \& Min, B. H. Low-intensity ultrasound (LIUS) as an innovative tool for chondrogenesis of mesenchymalstem cells (MSCs). Organogenesis 3, 74-78 (2007).

28. Schumann, D. et al. Treatment of human mesenchymal stem cells with pulsed low intensity ultrasound enhances the chondrogenic phenotype in vitro. Biorheology 43, 431-443 (2006).

29. Verdonk, P., V., R., Almqvist, F., Veys, E. M. \& Verbruggen, G. Fibrochondrogenic differentiation of human mesenchymal stem cells. J. Orthop. 1, 61-71 (2009).

30. $\mathrm{Yu}, \mathrm{B}$. et al. Simulated microgravity using a rotary cell culture system promotes chondrogenesis of human adipose-derived mesenchymal stem cells via the p38 MAPK pathway. Biochem. Biophys. Res. Commun. 414, 412-418 (2011).

31. Choi, J. W. et al. Mechanical stimulation by ultrasound enhances chondrogenic differentiation of mesenchymal stem cells in a fibrin-hyaluronic acid hydrogel. Artif. Organs 37, 648-655 (2013).

32. Sakao, K. et al. Induction of chondrogenic phenotype in synovium-derived progenitor cells by intermittent hydrostatic pressure. Osteoarthritis Cartil. 16 805-814 (2008).

33. Marsano, A. et al. Spontaneous in vivo chondrogenesis of bone marrow-derived mesenchymal progenitor cells by blocking vascular endothelial growth factor signaling. Stem Cells Transl. Med. https://doi.org/10.5966/sctm.2015-0321 (2016).

34. Unsworth, B. R. \& Lelkes, P. I. Growing tissues in microgravity. Nat. Med. 4, 901-907 (1998).

35. Freed, L. E. \& Vunjak-Novakovic, G. Microgravity tissue engineering. In Vitro Cell. Dev. Biol. Anim. 33, 381-385 (1997).

36. Freed, L. E. \& Vunjak-Novakovic, G. Cultivation of cell-polymer tissue constructs in simulated microgravity. Biotechnol. Bioeng. 46, 306-313 (1995).

37. Marsano, A. et al. Use of hydrodynamic forces to engineer cartilaginous tissues resembling the non-uniform structure and function of meniscus. Biomaterials 27, 5927-5934 (2006).

38. Dominici, M. et al. Minimal criteria for defining multipotent mesenchymal stromal cells. The International Society for Cellular Therapy position statement. Cytotherapy 8, 315-317 (2006).

39. Horwitz, E. M. et al. Clarification of the nomenclature for MSC: The International Society for Cellular Therapy position statement. Cytotherapy 7, 393-395 (2005).

40. Krawetz, R. J. et al. Synovial fluid progenitors expressing CD90+ from normal but not osteoarthritic joints undergo chondrogenic differentiation without micromass culture. PLoS One 7, e43616 (2012).

41. Acharya, C. et al. Enhanced chondrocyte proliferation and mesenchymal stromal cells chondrogenesis in coculture pellets mediate improved cartilage formation. J. Cell. Physiol. 227, 88-97 (2012).

42. Freed, L. E., Langer, R., Martin, I., Pellis, N. R. \& Vunjak-Novakovic, G. Tissue engineering of cartilage in space. Proc. Natl. Acad. Sci. USA 94, 13885-13890 (1997).

43. Mueller, M. B. et al. Hypertrophy in mesenchymal stem cell chondrogenesis: effect of TGF-beta isoforms and chondrogenic conditioning. Cells Tissues Organs 192, 158-166 (2010).

44. Steinert, A. et al. Hypertrophy is induced during the in vitro chondrogenic differentiation of human mesenchymal stem cells by bone morphogenetic protein2 and bone morphogenetic protein- 4 gene transfer. Arthritis Res. Ther. 11, R148 (2009).

45. Mueller, M. B. \& Tuan, R. S. Functional characterization of hypertrophy in chondrogenesis of human mesenchymal stem cells. Arthritis Rheum. 58, 1377-1388 (2008).

46. von der Mark, K. et al. Type X collagen synthesis in human osteoarthritic cartilage. Indication of chondrocyte hypertrophy. Arthritis Rheum. 35, 806-811 (1992).

47. van der Kraan, P. M. \& van den Berg, W. B. Chondrocyte hypertrophy and osteoarthritis: role in initiation and progression of cartilage degeneration? Osteoarthritis Cartil. 20, 223-232 (2012).

48. D'Angelo, M. et al. MMP-13 is induced during chondrocyte hypertrophy. J. Cell Biochem. 77, 678-693 (2000).

49. Leijten, J. C. et al. Gremlin 1, frizzled-related protein, and Dkk-1 are key regulators of human articular cartilage homeostasis. Arthritis Rheum. 64, 3302-3312 (2012).

50. Gazzerro, E. et al. Conditional deletion of gremlin causes a transient increase in bone formation and bone mass. J. Biol. Chem. 282, 31549-31557 (2007).

51. Martin, I., Muraglia, A., Campanile, G., Cancedda, R. \& Quarto, R. Fibroblast growth factor-2 supports ex vivo expansion and maintenance of osteogenic precursors from human bone marrow. Endocrinology 138, 4456-4462 (1997).

52. Bianchi, G. et al. Ex vivo enrichment of mesenchymal cell progenitors by fibroblast growth factor 2. Exp. Cell Res. 287, 98-105 (2003).

53. Solchaga, L. A., Penick, K., Goldberg, V. M., Caplan, A. I. \& Welter, J. F. Fibroblast growth factor-2 enhances proliferation and delays loss of chondrogenic potential 
in human adult bone-marrow-derived mesenchymal stem cells. Tissue Eng. Part $A$ 16, 1009-1019 (2010).

54. Di Maggio, N. et al. Fibroblast growth factor-2 maintains a niche-dependent population of self-renewing highly potent non-adherent mesenchymal progenitors through FGFR2c. Stem Cells 30, 1455-1464 (2012).

55. Bornes, T., Jomha, N., Mulet-Sierra, A. \& Adesida, A. Hypoxic culture of bone marrow-derived mesenchymal stromal stem cells differentially enhances in vitro chondrogenesis within cell-seeded collagen and hyaluronic acid porous scaffolds. Stem Cell Res. Ther. 6, 84 (2015).

Open Access This article is licensed under a Creative Commons Attribution 4.0 International License, which permits use, sharing, adaptation, distribution and reproduction in any medium or format, as long as you give appropriate credit to the original author(s) and the source, provide a link to the Creative Commons license, and indicate if changes were made. The images or other third party material in this article are included in the article's Creative Commons license, unless indicated otherwise in a credit line to the material. If material is not included in the article's Creative Commons license and your intended use is not permitted by statutory regulation or exceeds the permitted use, you will need to obtain permission directly from the copyright holder. To view a copy of this license, visit http://creativecommons. org/licenses/by/4.0/.

c) The Author(s) 2017 